\title{
Neuropeptides, récepteurs et satiété : la famille s'agrandit
}

Conséquence génétique et/ou inhérente au mode de vie, l'excès pondéral est une menace permanente pour l'organisme. Et pourtant, ce ne sont pas les «garde-fous» qui manquent. Ainsi, parmi les neuropeptides et peptides biologiques susceptibles d'être impliqués dans le contrôle de la prise alimentaire, plus d'une dizaine sont reconnus comme anorexigènes contre seulement quelques-uns orexigènes $\left(\mathrm{m} / \mathrm{s} n^{\circ} 1\right.$, vol. 13, p. 102), comme le neuropeptide Y (NPY) $\left(m / s n^{\circ} 10\right.$, vol. 12 , p. 1134), et la galanine $\left(\mathrm{m} / \mathrm{s}^{\circ} 5\right.$, vol. 12, p. 624). Ce réseau complexe de régulation neuropeptidergique de la fonction alimentaire s'enrichit aujourd'hui d'un nouvel intervenant anorexigène avec le récepteur BRS-3 (bombesin-receptor subtype-3), récepteur orphelin de ligand chez les mammifères, dont la fonction a été découverte lors de l'invalidation du gène [1]. Une stratégie utilisant des oligonucléotides antisens a permis de démontrer clairement la participation du récepteur hypothalamique NPY-Y5, un nouveau sous-type de récepteur NPY, dans le contrôle tonique du comportement alimentaire [2].

L'invalidation d'un gène permet, dans les situations les plus favorables, de confirmer, voire de découvrir, les véritables fonctions physiologiques de la protéine d'intérêt. Le récepteur BRS-3 apporte ici l'illustration même de cette stratégie. La bombésine, peptide de 14 acides aminés isolé initialement de la peau d'amphibien, outre une dizaine de peptides frères dans ces espèces, n'a que deux proches parents chez les mammifères, le GRP (gastrin-releasing peptide) et la neuromédine B. Tous deux se lient à des récepteurs distincts, l'un reconnaissant préférentiellement le GRP, l'autre, la neuromédine $B$. L'ADNc d'un troisième sous-type de récepteur de la bombésine, BRS-3, a été cloné récemment chez l'homme (par homologie avec le récepteur du GRP). Présent dans le testicule, le poumon et l'hypothalamus, ce récepteur ne dispose à ce jour ni de ligand spécifique, ni de fonction physiologique précise. Aussi, le phénotype des souris $B R S-3^{-/-}$, apparemment normal jusqu'à 16 semaines, a créé la surprise lorsqu'un excès pondéral a été décelé, maximal entre 27 et 34 semaines, clairement associé à une augmentation importante du tissu adipeux blanc. D'autres anomalies ont ensuite complété le tableau: augmentation de la pression sanguine, hyperinsulinémie, réduction de l'hormone de croissance plasmatique, diminution de la tolérance au glucose et à l'insuline. La plupart de ces anomalies qui témoignaient d'une perturbation du métabolisme des glucides et des lipides, étaient d'ailleurs caractéristiques des modèles animaux d'obésité. En outre, les souris $B R S-3^{-/-}$étaient non seulement hyperphagiques, mais, en cas de restriction alimentaire, perdaient moins de poids que les souris sauvages. Si leur activité locomotrice et la thermogenèse n'étaient pas altérées, le métabolisme basal était diminué. Enfin, l'hyperleptinémie détectée, reflet de l'excès de la masse adipeuse, suggérait que les souris $B R S-3^{-/-}$étaient insensibles à cette hormone. En l'absence d'altération morphologique au niveau de l'hypothalamus des souris $B R S-3^{-/-}$, la conclusion la plus probable était que le récepteur BRS-3 joue un rôle phy- siologique important dans la régulation de l'équilibre énergétique, action anorexigène relayée par l'intermédiaire de noyaux hypothalamiques spécifiques impliqués dans le comportement alimentaire. Reste désormais à identifier le ligand naturel!

A ce jour, le NPY est sans doute le neuropeptide orexigène le plus puissant de l'organisme, son administration centrale chronique pouvant conduire à une obésité par hyperphagie chez le rat $\left(\mathrm{m} / \mathrm{s} n^{\circ} 10\right.$, vol. 13 , p. 1202). A quels sous-types de récepteurs de NPY doit-on cette fonction vitale? En effet, plusieurs sous-types pharmacologiques de récepteurs de NPY ont été identifiés dans différents tissus dont l'hypothalamus, le plus récent désigné Y5 étant considéré, principalement sur des bases pharmacologiques, comme l'acteur régulateur princeps de cette fonction alimentaire $\left(\mathrm{m} / \mathrm{s} \mathrm{n}^{\circ} 10\right.$, vol. 12, p. 1134). Une stratégie qui a consisté à administrer in vivo des oligonucléotides antisens antipeptide NPY et antirécepteur NPY-Y5 ont finalement permis de confirmer définitivement cette hypothèse [2]. Ainsi, chez les rats traités par l'oligonucléotide antisens NPY, des études fonctionnelles ont montré que la prise alimentaire était diminuée de plus de $60 \%$ dans les deux heures qui suivent le jeûne, et de $20 \%$ environ dans les 18 heures. Cette situation semblait être la conséquence, d'une part, d'une diminution significative de la quantité d'aliments ingérée au cours du repas et de la durée du repas et, d'autre part, de l'allongement du délai entre les repas. Dans le cas de l'antisens anti-récepteur NPY-Y5, des modifications de même type, mais 
encore plus importantes, du comportement alimentaire ont été observées; la prise alimentaire spontanée, qu'elle soit stimulée par le NPY exogène, ou précédée d'un jeûne, était en effet plus réduite (réduction de $60 \%$ à $76 \%$ en 8 heures). La similitude des effets observés en présence des oligoantisens anti-NPY et antirécepteur Y5 a permis de suggérer que les récepteurs Y5 relaient en fait la quasi totalité des effets du NPY sur le comportement alimentaire. Le NPY et le récepteur Y5 semblent jouer un rôle important dans l'hyperphagie compensatoire qui suit une période de jeûne, mais également sur la prise alimentaire spontanée et son contrôle tonique. En outre, le rôle probable d'autres systèmes neuropeptidergiques comme la galanine ne doit pas être exclu. Les perspectives d'application de ces travaux pour une thérapie de l'obésité ne tar- deront probablement pas à se développer.

B.A.

1. Ohki-Hamazaki H, Watase K, Yamamoto K, Ogura H, Yamano M, Yamada K, Maeno H, Imaki J, Kikuyama S, Wada E, Wada K. Mice lacking bombesin receptor subtype-3 develop metabolic defects and obesity. Nature 1997; 390 : 166-9.

2. Schaffhauser AO, Stricker-Kongrad A, Brunner L, Cumin, F, Gerald C, Whitebread S, Criscione L, Hofbauer KG. Inhibition of food intake by neuropeptide Y Y5 receptor antisense oligodeoxynucleotides. Diabetes 1997; 46: 1792-8.

\section{BRÈVES}

La survie de la cellule $\beta$-pancréatique serait-elle une question de canal ? Les protéines Kir6.2 (canaux potassiques dits à rectification entrante), et SUR1 (récepteur des sulfonylurées de la famille des protéines membranaires liant l'ATP), constituent grâce à un assemblage multimérique le canal $\mathrm{K}^{+}$sensible à l'ATP $\left(\mathrm{K}_{(\mathrm{ATP})}\right)$ de la cellule $\beta$ pancréatique, impliqué dans le mécanisme de la sécrétion d'insuline $\left(\mathrm{m} / \mathrm{s} n^{\circ} 2\right.$, vol. 12, p. 251). Chez l'homme, la mutation du gène SUR1 peut conduire, par perte de l'activité du canal $\mathrm{K}_{\text {(ATP) }}$ à la forme familiale de l'hypoglycémie hyperinsulinique infantile $\left(m / s n^{\circ} 2\right.$, vol. 13, p. 276) [1]. Quelle est l'importance de Kir6.2 ? Pour répondre à la question, un groupe japonais a créé des souris transgéniques produisant en excès, dans la cellule $\beta$ pancréatique, la protéine
Kir6.2 rendue inactive par mutagenèse [2]. In vivo, le produit du transgène est supposé participer à la formation du tétramère de sousunités Kir6.2 et «titrer » la protéine Kir6.2 endogène, abolissant ainsi son activité de transport sélectif du $\mathrm{K}^{+}$. De fait, l'analyse des cellules $\beta$ pancréatiques des souris transgéniques montre que l'activité du $\mathrm{K}_{\text {(ATP) }}$ est extrêmement réduite; le potentiel de membrane basal et le calcium intracellulaire sont plus élevés que normalement. Conséquence probable de ces anomalies, la sécrétion d'insuline n'est plus du tout corrélée à la glycémie et les nouveau-nés sont hypoglycémiques. Plus tard, entre 2 et 4 semaines, apparaissent des anomalies à la fois morphologiques et fonctionnelles qui s'aggravent avec l'âge: une hyperglycémie basale, une faible sécrétion d'insuline en réponse au glucose, un nombre réduit de cellules $\beta$ pancréatiques, une localisation anormale des cellules à glucagon au centre de l'îlot et non plus à la périphérie, des îlots petits et déformés contenant, en outre, de nombreuses cellules apoptotiques. Scénario possible pour expliquer ce phénotype, la dépolarisation membranaire chronique liée à l'inactivation des canaux $\mathrm{K}_{(\mathrm{ATP})}$, conduirait, à long terme, à l'apoptose cellulaire à l'origine de l'hyperglycémie de l'adulte. Les canaux potassiques Kir6.2 apparaissent donc essentiels, non seulement au transport ionique mais aussi pour préserver la morphologie de l'îlot, et donc sa survie.

[1. Thuringer D, Cavero I. Med Sci 1997; 13: 1049-52.]

[2. Miki T, et al. Proc Natl Acad Sci USA 1997; 94: 11969-73.] 